University of Wollongong

Research Online

Faculty of Informatics - Papers (Archive)

Faculty of Engineering and Information

Sciences

January 2007

\title{
Generalized Bhaskar Rao Designs with Block Size 3 over Finite Abelian Groups
}

G. Ge

Zhejiang University, China

M. Grieg

Greig Consulting, North Vancouver, B.C., Canada

Jennifer Seberry

University of Wollongong, jennie@uow.edu.au

R. Seberry

Turramurra, Australia

Follow this and additional works at: https://ro.uow.edu.au/infopapers

Part of the Physical Sciences and Mathematics Commons

\section{Recommended Citation}

Ge, G.; Grieg, M.; Seberry, Jennifer; and Seberry, R.: Generalized Bhaskar Rao Designs with Block Size 3 over Finite Abelian Groups 2007.

https://ro.uow.edu.au/infopapers/596

Research Online is the open access institutional repository for the University of Wollongong. For further information contact the UOW Library: research-pubs@uow.edu.au 


\title{
Generalized Bhaskar Rao Designs with Block Size 3 over Finite Abelian Groups
}

\author{
Abstract \\ We show that if $\mathrm{G}$ is a finite Abelian group and the block size is 3 , then the necessary conditions for the \\ existence of a $(v ; 3 ; \lambda ; G)$ GBRD are sufficient. These necessary conditions include the usual necessary \\ conditions for the existence of the associated $(v ; 3 ; \lambda)$ BIBD plus $\lambda \equiv 0(\bmod |G|)$, plus some extra \\ conditions when $|G|$ is even, namely that the number of blocks be divisible by 4 and, if $v=3$ and the Sylow \\ 2 -subgroup of $\mathrm{G}$ is cyclic, then also $\lambda \equiv 0(\bmod 2|G|)$.

\section{Keywords} \\ Generalized Bhaskar Rao design, Cyclic group divisible designs \\ Disciplines \\ Physical Sciences and Mathematics

\section{Publication Details} \\ This article was originally published as Ge, G, Grieg, M, Seberry, J, \& Seberry, R, Generalized Bhaskar Rao \\ Designs with Block Size 3 over Finite Abelian Groups, Graphs and Combinatorics, 23(3), 2007, 271-290. \\ The original publication is available at www.springerlink.com.
}




\title{
Generalized Bhaskar Rao Designs with Block Size 3 over Finite Abelian Groups
}

\author{
Gennian $\mathrm{Ge}^{1}$, Malcolm Greig ${ }^{2}$, Jennifer Seberry ${ }^{3}$, Ralph Seberry ${ }^{4}$ \\ 1 Department of Mathematics, Zhejiang University, Hangzhou 310027, Zhejiang, P. R. China. \\ e-mail: gnge@zju.edu.cn \\ ${ }^{2}$ Greig Consulting, 317-130 Eleventh St. East, North Vancouver, B.C., V7L 4R3, Canada. \\ e-mail: greig@sfu.ca \\ 3 Centre for Computer Security Research, SITACS, University of Wollongong, Wollongong, \\ NSW 2522, Australia. e-mail: j.seberry@uow.edu.au \\ 47 Leeds Place, Turramurra, NSW 2074, Australia.
}

\begin{abstract}
We show that if $G$ is a finite Abelian group and the block size is 3, then the necessary conditions for the existence of a $(v, 3, \lambda ; G)$ GBRD are sufficient. These necessary conditions include the usual necessary conditions for the existence of the associated $(v, 3, \lambda)$ BIBD plus $\lambda \equiv 0(\bmod |G|)$, plus some extra conditions when $|G|$ is even, namely that the number of blocks be divisible by 4 and, if $v=3$ and the Sylow 2-subgroup of $G$ is cyclic, then also $\lambda \equiv 0$ $(\bmod 2|G|)$.
\end{abstract}

Key words. Generalized Bhaskar Rao design, Cyclic group divisible designs.

\section{Introduction}

Our aim in this article is to show that the necessary conditions for a Generalized Bhaskar Rao Design with block size 3 over any finite Abelian group are sufficient.

A Generalized Bhaskar Rao Designs over the group $G$ is formed from a balanced incomplete block design. To form the $(v, k, \lambda ; G)$ GBRD we take the incidence matrix of a $(v, k, \lambda)$ BIBD and replace the non-zero elements by elements from the group $G$. Suppose we replace the incidence of the point $a$ (resp. $b$ ) in the $i$-th block by the group element $a_{i}$ (resp. $b_{i}$ ). Then the replacement must be done in such a way that the list $a_{i} b_{i}^{-1}$ (where $i$ runs through all blocks with both $a$ and $b$ present) contains every element of $G$ exactly $\lambda /|G|$ times.

Theorem 1. Let $G$ be a finite Abelian group. The necessary conditions for the existence of $a(v, 3, \lambda ; G)$ GBRD for $v>3$ are:

$$
\begin{aligned}
& \lambda \equiv 0(\bmod |G|) \\
& \lambda(v-1) \equiv 0(\bmod 2) \\
& \lambda v(v-1) \equiv\left\{\begin{array}{llll}
0 & (\bmod 6) & \text { if }|G| \equiv 1 & (\bmod 2) \\
0 & (\bmod 24) & \text { if }|G| \equiv 0 & (\bmod 2)
\end{array}\right.
\end{aligned}
$$


When $v=3$, we always have the restriction (1), but if the Sylow 2-subgroup of $G$ is non-trivial and cyclic, then for a $(3,3, \lambda ; G) G B R D$ we also require:

$$
\lambda \equiv 0(\bmod 2|G|)
$$

The object of this article is to establish Theorem 2 .

Theorem 2. Let $G$ be a finite Abelian group. The necessary conditions for the existence of a $(v, 3, \lambda ; G)$ GBRD given in Theorem 1 are sufficient.

The necessary conditions (discussed in the next section) are known to be sufficient for a $(v, 3, \lambda ; G)$ GBRD over a number of groups. The classic case $G=Z_{2}$ was solved by Seberry [24]. The elementary Abelian groups not divisible by 6 were solved by Lam and Seberry [19] and the remaining elementary Abelian groups by Seberry [25]. Palmer and Seberry [22] solved the non-Abelian groups $D_{3}, D_{4}, D_{6}$ and $Q_{4}$ as well as the (nonelementary) Abelian $Z_{2} \times Z_{4}$. Combe, Palmer and Unger [9] have solved $A_{4}$, the alternating group of order 12, and Abel, Combe and Palmer [2] have since solved the remaining groups of order 12. Abel, Combe and Palmer [3] have also solved the existence problem for arbitrary dihedral groups. Some (non-elementary) Abelian groups have been studied: $Z_{4}$ was solved, apart from two open exceptions, by de Launey, Sarvate and Seberry [11], and $Z_{8}$ was solved by Seberry [26]. Shen's labelled designs (e.g., [28]) are also GBRDs over the cyclic groups presented in a less compact notation.

There is a close link between $(v, k, \lambda)$ GBRDs and group divisible designs, specifically $(k, \lambda /|G|)$ GDDs of type $|G|^{v}$. (For a definition of standard designs such as BIBDs, GDDs, TDs and PBDs, see e.g., [5].) This was one of the early motivations for studying GBRDs $[24,27,29]$. In fact if we replace each signed element in the GBRD incidence matrix by the corresponding right-regular $|G|$ by $|G|$ permutation matrix and each zero incidence by a $|G|$ by $|G|$ matrix of zeros, then we get the incidence matrix of a $(k, \lambda /|G|)$ GDD of type $|G|^{v}$.

Working with this GDD form, the necessary conditions for the existence of a $\left(v, 3, n ; Z_{n}\right)$ GBRD were shown to be sufficient by Jiang [18] for $n$ a multiple of 6 , and for the other $n$ s by Gallant, Jiang and Ling [14]. However, Jiang's solution takes a fair amount of work to translate into a design and Gallant et al. do not give explicit solutions in all cases, giving instead a heuristic algorithm with which they state they quickly found appropriate solutions in every case but one, for which case they give two solutions, an invalid one [14, pp. 102] and a valid one as a part of [14, Lemma 2.5].

We discuss the necessary conditions in Section 2. In Sections 3 and 4 we give our composition methods, and in Section 6 we give some incomplete arrays (or holey difference matrices (HDMs for short)) which we use there to solve the $v=3$ case. The remaining sections deal mostly with small numbers of invariants of various Sylow subgroups and, in the final Section 10, we establish the sufficiency of the necessary conditions for the general case. Our main asymptotic construction is Theorem 18, which reduces the problem to the construction of a small number of designs for any given abelian group. Here we can rely on mostly known PBD and BIBD results (in Theorem 19 we do establish a new PBD basis result for $K=\{4,9,12\}$ ). However, we do have to provide the small designs, as well as some improved composition methods to solve the problem. A few designs are given in the main text, but most of the component designs we need are given in the Appendix. 


\section{The Necessary Conditions}

In this section we discuss the necessary conditions. The necessary conditions for the existence of a $(v, k, \lambda ; G)$ GBRD must obviously include the necessary conditions for the existence of a $(v, k, \lambda) \mathrm{BIBD}$ and that $\lambda /|G|$ be integral, i.e., the necessary conditions for the existence of a $(v, k, \lambda ; G)$ GBRD include:

$$
\begin{aligned}
\lambda & \equiv 0(\bmod |G|) \\
\lambda(v-1) & \equiv 0(\bmod (k-1)) \\
\lambda v(v-1) & \equiv 0(\bmod (k(k-1))) .
\end{aligned}
$$

Now, when $k=3$, Conditions (6) with (7) are known to be sufficient for the existence of a $(v, k, \lambda)$ BIBD [17]. However, there are some extra restrictions on GBRDs for some groups, especially when $v=k$.

Theorem 3. (Drake [13, Theorem 1.10]) Let $G$ be a finite group of even order with a cyclic Sylow 2-subgroup, and let $k \geq 3$. If $\lambda /|G|$ is odd, then there is no $(k, k, \lambda ; G)$ GBRD.

The following result is also well-known, but usually phrased in terms of difference matrices using a different notation (e.g., [5, Lemma VIII.3.1]).

Theorem 4. If $\lambda<k$, then there is no $(k, k, \lambda ; G) G B R D$.

There is one further condition known for $G=Z_{2}$, which in the case of $k=3$ reduces to:

$$
b \equiv 0(\bmod 4)
$$

where $b$ is the number of blocks in the GBRD. The extension to general $k$ is given in [24]. The restriction has applicability to other groups however.

Theorem 5. (Gibbons and Mathon [15, Theorem 2]) Let $N$ be a normal subgroup of $G$, and suppose $a(v, k, \lambda ; G) G B R D$ exists. Then $a(v, k, \lambda ; G / N) G B R D$ exists.

As examples of how Theorem 5 extends Condition (8), consider $n$ odd, and the normal subgroup $Z_{n}$ in $Z_{2 n}$. (Similar observations hold, e.g., for $Z_{n}$ in the dihedral group $D_{n}$ of order $2 n$ with $n$ odd.) The group $Z_{2 n} / Z_{n}$ amounts to the group $Z_{2}$ with the elements labelled as "odd" or "even". In a $\left(v, 3, \lambda ; Z_{2 n} / Z_{n}\right)$ GBRD, for any pair of points we need the number of mixed parity signings to be equal to the number of same parity pairings, but a block can only contribute either 3 same parity, or 1 same and 2 mixed parity pairings. Consequently, in the design each block of the former (3 same) type must be balanced by 3 of the latter, hence the restriction that $b \equiv 0(\bmod 4)$ must apply here too.

So when might Condition (8) be a restriction? The number of blocks in a $(v, 3, \lambda)$ BIBD is $\lambda\left(\begin{array}{l}v \\ 2\end{array}\right) / 3$, and clearly we will assume $G$ has even order, so $\lambda$ is even by Condition (5). If $\lambda \equiv 0(\bmod 4)$, then Condition (8) will be satisfied, so it is only an additional restriction if $|G| \equiv 2(\bmod 4)$ and $\lambda \equiv 2(\bmod 4)$ and $v \equiv 2,3(\bmod 4)$.

The necessary conditions were summarized earlier in Theorem 1. 


\section{Some Structural Results}

The structure of finite Abelian groups is known (see e.g., [23, Theorem 4.2.6]).

Theorem 6. (Frobenius-Stickleberger) An Abelian group, $G$, is finite if and only if it is a direct product of finitely many cyclic groups with prime power orders. (We will call these cyclic groups the invariants of $G$.)

Remark 7. Normally, the invariants of $G$ are considered to be the orders of the cyclic factor groups, rather than the cyclic factor groups themselves (see, e.g., [16, Section 3.3]), but our terminology should cause little confusion.

Remark 8. It will occasionally be convenient to characterize the trivial single element subgroup as $Z_{1}$.

Remark 9. If $\operatorname{gcd}(m, n)=1$, then the group $Z_{m n}$ is isomorphic to the group $Z_{m} \times Z_{n}$. The invertible mapping $(x) \mapsto(x(\bmod m), x(\bmod n))$ relates the elements of the isomorphs. We will find it convenient in some examples to use the group $Z_{m n}$.

Often a GDD of type $g^{v}$ will be given as a difference family over some group of order $g v$. When this factors as the direct product of a group $G$ of order $g$ and a group $V$ of order $v$ and the groups of the GDD are the sets $G \times\{x\}$ for $x \in V$, then there is no difficulty in converting this difference family into the $(v, k, \lambda ; G)$ GBRD form.

The next two examples use Bose's affine relative difference set over $Z_{q^{2}-1}$ [6]. These GDDs correspond to the punctured $\operatorname{AG}(2, q)$, and can be formed by taking the discrete logarithm in $\operatorname{GF}\left(q^{2}\right)$ of $(1+a x)$ for $a \in G F(q)$. Note that $\operatorname{GF}\left(q^{2}\right)$ is the extension field over $\mathrm{GF}(q)$. Here, the logarithms are taken with respect to a root $x$ of $x^{2}=y x+y$ in $\mathrm{GF}(16)$ where $y$ is a root of the primitive polynomial $y^{2}=y+1$ in $\operatorname{GF}(4)$, and $x^{2}=y^{4} x+y^{5}$ in $\mathrm{GF}(81)$ where $y$ is a root of the primitive polynomial $y^{2}=y+1$ in $\mathrm{GF}(9)$.

Example 10. A $(4,1)$ GDD of type $3^{5}$ over $Z_{15}$ is given by the difference set $(0,12,8,14)$. The mapping given in Remark 9 gives the corresponding $\left(5,4,3 ; Z_{3}\right)$ GBRD form as $\left(0_{0}, 2_{0}, 3_{2}, 4_{2}\right)$ which is developed over $Z_{5}$, with the subscripts (i.e., the signings) fixed.

Table 1. The signed incidence matrix of a $\left(10,9,8 ; Z_{8}\right)$ GBRD

$\begin{array}{llllllllll}0 & 5 & 7 & 5 & 5 & 6 & 1 & 0 & 4 & \\ & 0 & 5 & 7 & 5 & 5 & 6 & 1 & 0 & 4 \\ 3 & & 0 & 5 & 7 & 5 & 5 & 6 & 1 & 0 \\ 7 & 3 & & 0 & 5 & 7 & 5 & 5 & 6 & 1 \\ 0 & 7 & 3 & & 0 & 5 & 7 & 5 & 5 & 6 \\ 5 & 0 & 7 & 3 & & 0 & 5 & 7 & 5 & 5 \\ 4 & 5 & 0 & 7 & 3 & & 0 & 5 & 7 & 5 \\ 4 & 4 & 5 & 0 & 7 & 3 & & 0 & 5 & 7 \\ 6 & 4 & 4 & 5 & 0 & 7 & 3 & & 0 & 5 \\ 4 & 6 & 4 & 4 & 5 & 0 & 7 & 3 & & 0\end{array}$


Example 11. A $(9,1)$ GDD of type $8^{10}$ is given by the difference set

$$
(0,49,47,73,46,55,68,4,32)
$$

which is developed over $Z_{80}$. The groups are points with the same residue modulo 10 . In order to convert this GDD to a $\left(10,9,8 ; Z_{8}\right)$ GBRD, we take the first 10 translates of the difference set, generating 90 points in sets of 9 , and for each point, say $x$, we convert to a signed point $y_{a}$ by the rule $y=x(\bmod 10)$ and $a=\lfloor x / 10\rfloor$. We give this design as a signed incidence matrix in Table 1.

This translation method also works for the difference set in Example 10, but the GBRD generated, although isomorphic, does not have such a nice representation as the one we gave in Example 10.

In some respects, this construction can be regarded as an inversion of the construction we use in Theorem 16.

In Section 1 we noted that $(v, k, \lambda ; G)$ GBRDs can be converted into $(k, \lambda /|G|)$ GDDs of type $|G|^{v}$. We now need an intermediate level conversion: specifically, of a $(v, k, \lambda ; G \times H)$ GBRD into a $(k, \lambda /|G|)$ GDD of type $|G|^{v}$ that is signed over $H$.

Theorem 12. If a $(v, k, \lambda ; G \times H) G B R D$ exists, then there exists a $(k, \lambda /|G|) G D D$ of type $|G|^{v}$ that is signed over $H$. We will denote this resultant design as a $(k, \mu ; H)$ GBRGDD of type $|G|^{v}$, where $\mu=\lambda /|G|$.

Proof. In the signed incidence matrix of the GBRD, replace every instance of an element signed by $(g, h)$ by the $|G|$ by $|G|$ permutation matrix representation of $g$, and sign every non-zero element with $h$, and replace every zero entry in the incidence matrix by a $|G|$ by $|G|$ matrix of zeros.

\section{Methods}

In this section we will present several product theorems which form the basis for our recursive approach to the existence problem.

Our first product theorem is a slightly improved version of [19, Theorem 2.1].

Theorem 13. If there exists a $(v, k, \lambda ; G) G B R D$, a $T D(k, u), a(u+w, k, \mu ; G) G B R D$, and $a(u+w, k, \mu ; G)$ GBRD missing $a(w, k, \mu ; G)$ GBRD subdesign, then $a$ (uv + $w, k, \mu \lambda ; G)$ GBRD exists.

Proof. We start with the design on $v$ points, and give all points a weight of $u$ in a variant of Wilson's Fundamental Construction where the signing of the $(v, k, \lambda)$ GBRD is inherited by the newly generated points, then use the TD as the ingredient design to get a signed GDD of type $u^{v}$. We adjoin $w$ new points, then fill $v-1$ of the groups using the incomplete $(u+w, k, \mu ; G)$ GBRD, aligning the missing subdesign on the adjoined points. Finally, we use the complete $(u+w, k, \mu ; G)$ GBRD to fill in the last group plus the adjoined points.

Remark 14. Every $(u+w, k, \mu ; G)$ GBRD is missing a $(w, k, \mu ; G)$ GBRD subdesign if $w=0$ or 1: these trivial subdesigns contain no blocks.

The following is an obvious generalization of theorems given by Gibbons and Mathon [15, Theorem 1] and Lam and Seberry [19, Theorem 3.3]. 
Theorem 15. If there exists a $(v, K, \lambda ; G) G B R D$ and $a(k, j, \mu ; H) G B R D$ exists for each $k \in K$, then $a(v, j, \mu \lambda ; G \times H) G B R D$ exists.

Proof. When $G$ and $H$ are just the trivial group $Z_{1}=\{0\}$, then this theorem just amounts to the standard "breaking the blocks" construction, where we take the $i$-th block of the set $\mathcal{B}_{G}$, say $B_{i}=\left(b_{1}, b_{2}, \ldots, b_{k}\right)$, and form the $(k, j, \mu)$ PBD on these $k$ points, and finally juxtapose all these designs for our resultant design. So now we just have to deal with the signing. Suppose a point of $B_{i}$ receives a signing of $g$, and in the $\ell$-th block of the design breaking it, that point receives a signing of $h$, then the signing we now assign to that point is $f=g \times h$.

Lam and Seberry [19, Theorem 3.1] give a construction which allows us to combine a $\left(k, k, n ; Z_{n}\right)$ GBRD and a $\left(k, k, m ; Z_{m}\right)$ GBRD into a $\left(k, k, m n ; Z_{m n}\right)$ GBRD. We give a generalization of this construction, which is a special case of a broader generalization of Palmer's [21, Theorem 2.1].

Theorem 16. Let $G=Z_{n_{1}} \times Z_{n_{2}} \times \cdots \times Z_{n_{t}}$ and $H=Z_{m_{1}} \times Z_{m_{2}} \times \cdots \times Z_{m_{t}}$. Note that we will allow the possibilities that one or more $n_{i}=1$, and similarly one or more $m_{i}=1$. If there exists a $(v, K, \lambda ; G) G B R D$ and $a(k, j, \mu ; H)$ GBRD exists for each $k \in K$, then $a(v, j, \mu \lambda ; F) G B R D$ exists, where $F=Z_{m_{1} n_{1}} \times Z_{m_{2} n_{2}} \times \cdots \times Z_{m_{t} n_{t}}$.

Proof. When $G, H$ and $F$ are just the trivial group $Z_{1}=\{0\}$, then this theorem just amounts to the standard "breaking the blocks" construction, where we take the $i$-th block of the set $\mathcal{B}_{G}$, say $B_{i}=\left(b_{1}, b_{2}, \ldots, b_{k}\right)$, and form the $(k, j, \mu)$ PBD on these $k$ points, and finally juxtapose all these designs for our resultant design. So now we just have to deal with the signing. Suppose a point of $B_{i}$ receives a signing of $g_{1} \times g_{2} \times \cdots \times g_{t}$, and in the $\ell$-th block of the design breaking it, that point receives a signing of $h_{1} \times h_{2} \times \cdots \times h_{t}$, then the signing we now assign to that point is $f_{1} \times f_{2} \times \cdots \times f_{t}$, where $f_{i}=g_{i}+n_{i} h_{i}$.

Remark 17. If $G=Z_{n} \times Z_{1}$ and $H=Z_{1} \times Z_{m}$, so $F=Z_{n} \times Z_{m}$ in Theorem 16, then Theorem 16 reduces to Theorem 15 .

One of our main tools will be the following special case of Theorem 15, obtained by setting $G$ (in Theorem 15) to be the trivial group.

Theorem 18. If there exists a $(v, K, \lambda) P B D$ and, for each $k \in K, a(k, j, \mu ; G) G B R D$ exists, then a $(v, j, \mu \lambda ; G) G B R D$ exists.

Application of Theorem 18 to the hypothesized PBD means we only have to deal with the $v$ s in some basis set for any group. We summarize these for $\lambda=|G|$ in Table 2. (The PBD basis sets in Table 2 can mostly be found in [4], updated by [20]. There is one new PBD result, given in Theorem 19.)

Theorem 19. $A(v,\{4,9,12\}, 1) P B D$ exists when $v \equiv 0,1,4,9(\bmod 12)$ with the definite exception of $v \in\{21,24\}$.

Proof. A $(v,\{4,9\}, 1)$ PBD exists if $v \equiv 0,1,4,9(\bmod 12)$ and $v \notin\{12,21,24,48\}$, except possibly when $v \in\{60,69,84,93,96,192\}$ by a result of Mullin et al. [20]. Brouwer et al. [8] showed a 4-GDD of type $12^{n}$ is known for all $n \geq 4$, so our result for the multiples of 12 follows by filling these GDDs. For $v=69$ and 93, we truncate to size three the largest group of a 5-GDD of type $4^{6}$ or $4^{7} 8^{1}$ (where the latter is formed by completing a 4-RGDD), then give all points a weight of three in Wilson's fundamental construction (a 4 -GDD of type $3^{5}$ and a $\operatorname{TD}(4,3)$ provide the ingredients), and finally fill the groups of the resulting 4-GDD. 
Table 2. Necessary conditions for $\lambda=|G|$

\begin{tabular}{cccl}
$|G| \bmod 12$ & Sylow 2-s.g. & $v$ condition & PBD basis \\
\hline $1,5,7,11$ & Trivial & $1,3(\bmod 6)$ & 3 \\
2,10 & Cyclic & $0,1,4,9(\bmod 12)$ & $4,9,12,21,24$ \\
3,9 & Trivial & $1(\bmod 2)$ & 3,5 \\
4,8 & Cyclic & $0,1(\bmod 3), v>3$ & $4,6,7,9,10,12,15,18,19,24,27$ \\
4,8 & Non-cyclic & $0,1(\bmod 3)$ & $3,4,6$ \\
6 & Cyclic & $0,1(\bmod 4)$ & $4,5,8,9,12$ \\
0 & Cyclic & $v>3$ & $4-12,14,15,18,19,23$ \\
0 & Non-cyclic & $v \geq 3$ & $3,4,5,6,8$
\end{tabular}

\section{Odd Invariants}

In this section we will look at $G=Z_{g}$ with $g$ odd. There is a well-known general construction available.

Lemma 20. $A\left(3,3, t ; Z_{t}\right)$ GBRD exists for any odd $t \geq 1$.

Proof. The design on $I_{3}$ is given by $\left(0_{0}, 1_{i}, 2_{2 i}\right)$ where $i=0,1, \ldots, t-1$.

Theorem 21. If $v \equiv 1,3(\bmod 6)$ and $|G|$ is odd, then $a(v, 3,|G| ; G)$ GBRD exists.

Proof. We may start with a $(v, 3,1)$ BIBD, and apply Theorem 15 for each invariant, $G_{i}$, where the $\left(3,3,\left|G_{i}\right| ; G_{i}\right)$ GBRD is given by Lemma 20 , and where $G=G_{1} \times \cdots \times G_{t}$ is the decomposition of $G$ as a product of cyclic groups.

\subsection{One Invariant Divisible by Three}

In this subsection we will look at $G=Z_{g}$ with $g$ being a power of 3 .

Lemma 22. If $v \equiv 1(\bmod 2)$, then $a\left(v, 3,3 ; Z_{3}\right) G B R D$ exists.

Proof. Let $K=\{3,5\}$. It suffices to establish the existence of a $\left(v, 3,3 ; Z_{3}\right)$ GBRD for $v \in K$, since for other $v$ s a $(v, K, 1)$ PBD exists by Table 2 , so then we can use Theorem 18 for our result. We have given $\left(v, 3,3 ; Z_{3}\right)$ GBRDs for $v=3$ and 5 in Lemma 20 and Example 50.

Theorem 23. If $v \equiv 1(\bmod 2)$, then $a\left(v, 3,3 t ; Z_{3 t}\right) G B R D$ exists if $t$ is a power of 3 .

Proof. Take the $\left(v, 3,3 ; Z_{3}\right)$ GBRD given by Lemma 22 and the $\left(3,3, t ; Z_{t}\right)$ GBRD given by Lemma 20 . We may then apply Theorem 16 to these to get our result.

\section{The case $v=3$ and Holey Difference Matrices}

Now we have dealt with $v=3$ for an odd invariant, and we know just one even invariant is impossible. We will now look at the case where $G$ consists of two or three even invariants, and then extend to the general case. Difference matrices and HDMs are used to accomplish this, but we will also find other uses for some of the HDMs we construct here. Holey quasidifference matrices are normally defined in more generality than we need. For example, 
in Abel et al.'s terminology (see [1]), all the HDMs we consider are $(|G|,|H|, 0,1,0 ; k)$ HQDMs, where $H$ is a normal subgroup of $G$, and the holes which partition $G$ are given by the cosets of $H$. We will refer to our HQDMs as $(G, H ; k)$ HDMs. They satisfy the property that for any two distinct rows $i$ and $j$, the products $d_{i t} * d_{j t}^{-1}$ yield every element of $G$ once, except that the elements of $H$ do not occur. A $(G, H ; k)$ HDM has size $k$ by $|G|-|H|$.

Theorem 24. $A\left(3,3,4 s t ; Z_{2 s} \times Z_{2 t}\right)$ GBRD exists when $1 \leq s \leq t$ are both powers of two.

Proof. If $4 \leq s \leq t$, we may form a direct product of the $\left(Z_{2 s}, Z_{2} ; 3\right) \mathrm{HDM}$ and the $\left(Z_{2 t}, Z_{2} ; 3\right) \mathrm{HDM}$ given in Example 41. Suppose these arrays are $(0, i, f(i))^{T}$ and $(0, j, f(j))^{T}$. Then $((0,0),(i, j),(f(i), f(j)))^{T}$ is the product which we form for all possible pairs of $i$ with $j$. We next adjoin the $\left(Z_{2} \times Z_{2 t}, Z_{2} \times Z_{2} ; 3\right) \mathrm{HDM}$ given in Example 43, with the normal subgroup being $\langle 0, s\rangle \times\langle 0, t\rangle$, then adjoin the $\left(Z_{2 s} \times Z_{2}, Z_{2} \times Z_{2} ; 3\right) \mathrm{HDM}$ given in Example 43, with the normal subgroup again being $\langle 0, s\rangle \times\langle 0, t\rangle$. Finally, we adjoin a DM on the normal $Z_{2} \times Z_{2}$ subgroup.

If $s=1$ and $t \geq 4,\left(Z_{2} \times Z_{2 t}, Z_{2} \times Z_{2} ; 3\right) \mathrm{HDM}$ given in Example 43, with the normal subgroup being $\langle 0, s\rangle \times\langle 0, t\rangle$, then adjoin the DM on the normal $Z_{2} \times Z_{2}$ subgroup.

If $s=1$ and $t \leq 2$, the design is given in Example 39 .

If $s=2$ and $t \geq s$, then we may take the product of a $\left(3,3,4 ; Z_{2} \times Z_{2}\right)$ GBRD and a $\left(3,3,4 ; Z_{2} \times Z_{t}\right)$ GBRD design using Theorem 16 .

Theorem 25. $A\left(3,3,8 r s t ; Z_{2 r} \times Z_{2 s} \times Z_{2 t}\right)$ GBRD exists when $1 \leq r \leq s \leq t$ are all powers of two.

Proof. If $t \geq 2$, we can take the product of a $\left(3,3,4 r ; Z_{2 r} \times Z_{2} \times Z_{1}\right)$ GBRD and a $\left(3,3,2 s t ; Z_{1} \times Z_{t} \times Z_{2 s}\right)$ GBRD design using Theorem 16 .

If $t=1$, then $r=s=1$ and the design is given in Example 39 .

Theorem 26. If the Sylow 2-subgroup is either trivial or non-cyclic, then a $(3,3,|G| ; G)$ GBRD exists.

Proof. This follows using the $\left(3,3,\left|G_{i}\right| ; G_{i}\right)$ GBRDs given by Lemma 20 and Theorems 24 and 25 and repeated application of Theorem 15 to form a $(3,3,|G| ; G)$ GBRD with $G=$ $G_{1} \times \cdots \times G_{t}$.

There are two particular designs we need to highlight.

Corollary 27. If $t \geq 1$ is a power of 2 , then a $(3,3,|G| ; G)$ GBRD exists for $G=Z_{2} \times$ $Z_{2} \times Z_{2 t}$ and for $G=Z_{3} \times Z_{2} \times Z_{2 t}$.

\section{A Cyclic Sylow 2-Subgroup}

In this section we will look at $G=Z_{g}$ with $g$ being a power of 2 . There is no $(3,3,|G| ; G)$ GBRD for this class, and the case $Z_{2}$ is rather different from the larger cyclic groups because of the restriction that the number of blocks must be a multiple of 4 . There are a couple of general constructions available.

Lemma 28. If $t \geq 1$ is a power of two, then a $\left(12,3,2 t ; Z_{2 t}\right)$ GBRD exists, and an $\left(18,3,2 t ; Z_{4 t}\right) G B R D$ exists. 
Proof. By Corollary 27, we have a $\left(3,3,2 t|H| ; H \times Z_{2 t}\right)$ GBRD where $H=Z_{2} \times Z_{2}$ or $H=Z_{2} \times Z_{3}$, so by Theorem 12 , a $\left(3,2 t ; Z_{2 t}\right)$ GBRGDD of type $|H|^{3}$ exists for $|H|=4$ and 6 . We may then fill the groups by adjoining three $\left(|H|, 3,2 t ; Z_{2 t}\right.$ ) GBRDs (given in Examples 48 or 51) defined on the three groups for our GBRD.

\subsection{The Case $Z_{2}$}

Theorem 29. (Seberry [24]) If $v \equiv 0,1,4,9(\bmod 12)$, then $a\left(v, 3,2 ; Z_{2}\right)$ GBRD exists.

Proof. Let $K=\{4,9,12,21,24\}$. It will suffice to establish the existence of a $(v, 3,|G| ; G)$ GBRD for $v \in K$, since for other $v$ s a $(v, K, 1)$ PBD exists by Theorem 19, so then we can use Theorem 18 for our result.

We have given $\left(v, 3,2 ; Z_{2}\right)$ GBRDs for $v=4,9,12$ and 21 in Examples 48, 53, Lemma 28 and Example 58 (noting for $v=9$ and 21 that the missing subdesign on 4 points exists). For $v=24$ we may use Theorem 13 , noting that $24=4 \cdot(9-4)+4$.

\subsection{The Case $Z_{4 s}$}

Theorem 30. Let $t \geq 2$ be a power of 2 and $v \equiv 0,1(\bmod 3)$. If $v>3$, then a $\left(v, 3,2 t ; Z_{2 t}\right) G B R D$ exists.

Proof. Let $K=\{4,6,7,9,10,12,15,18,19,24,27\}$. It suffices to establish the existence of a $(v, 3,|G| ; G)$ GBRD for $v \in K$, since for other $v$ s a $(v, K, 1)$ PBD exists by Table 2 , so then we can use Theorem 18 for our result.

For $v=4$ and $v=6$ the GBRD is given by Examples 48 and 51 . For $v=7$ we can take the $\left(7,4,2 ; Z_{2}\right)$ GBRD given in Example 44 and use Theorem 16 to break the blocks with the $\left(4,3, t ; Z_{t}\right)$ GBRD given by Example 48 . For $v=9$ with $2 t=4$, the GBRD is given in Example 54. For the larger groups with $v=9$ we can use a construction of Bowler et al. [7, Lemma 2.3], so for $v=9$ with $t \geq 4$, we take a $(9,3,1)$ BIBD and replace every block by the $\left(Z_{2 t}, Z_{2} ; 3\right)$ HDM given in Example 42 , and finally adjoin the blocks of a $\left(9,3,2 ; Z_{2}\right)$ GBRD formed on the missing subdesign. For $v=10$ with $2 t=4$, the GBRD is given in Example 55. For $v=10$ with $2 t=8 s \geq 8$, we can take the $\left(10,9,8 ; Z_{8}\right)$ GBRD given in Example 11, and use the $\left(9,3, s ; Z_{s}\right.$ ) GBRD (constructed just above if $s \geq 4$, or in Theorem 29 if $s=2$ or else is given by a constantly signed BIBD if $s=1$ ) and thence our design follows by breaking the blocks via Theorem 16. For $v=12$ or 18, the GBRDs are given by Lemma 28. For $v=15$ or 27 , let $s=4$ if $2 t$ is a power of 4 and $s=8$ otherwise. A $\left(v, 3, s ; Z_{s}\right)$ GBRD is given in Examples 56, 57, 59 and 60 . For $2 t>4 s$, we take the $\left(Z_{2 t}, Z_{t / 2} ; 3\right)$ HDM given in Example 42 . By repeatedly augmenting this array with similar arrays defined on the normal subgroup, we eventually arrive at a $\left(Z_{2 t}, Z_{s} ; 3\right)$ HDM. We now take a $(v, 3,1)$ BIBD and replace every block by this augmented HDM defined on the points of that block, and then adjoin the blocks of a $\left(v, 3, s ; Z_{s}\right)$ GBRD formed on the normal subgroup. Finally, for $v=19$ and 24, we can apply Theorem 13, noting that $19=6 \cdot(4-1)+1$ and $24=6 \cdot 4$.

\section{Multiples of Six with a Cyclic Sylow 2-Subgroup}

In this section we will consider groups of the form $G=Z_{3} \times Z_{2 t}$ where $t \geq 1$ is a power of 2 . 
Theorem 31. Let $G=Z_{3} \times Z_{2 t}$ where $t \geq 1$ is a power of 2 . If $v \equiv 0,1(\bmod 4)$, then a $(v, 3,6 t ; G)$ GBRD exists.

Proof. Let $K=\{4,5,8,9,12\}$. It will suffice to establish the existence of a $(v, 3,|G| ; G)$ GBRD for $v \in K$, since for other $v$ s a $(v, K, 1)$ PBD exists by Table 2 , so then we can use Theorem 18 for our result.

A $\left(v, 4,3 ; Z_{3}\right)$ is given for $v=8,9$ and 12 in Example 45, and for $v=5$ in Example 10. We may use the $\left(4,3,2 t ; Z_{2 t}\right)$ GBRD of Example 48 with these designs in the direct product construction of Theorem 15 to give $\left(v, 3,6 t ; Z_{3} \times Z_{2 t}\right)$ GBRDs for these $v$ s. For $v=4$, we may use the $\left(3,3,3 s ; Z_{3}\right)$ GBRD of Lemma 20 with the $\left(4,3,2 t ; Z_{2 t}\right)$ GBRD of Example 48 in the direct product construction of Theorem 15 to give a $\left(4,3,6 t ; Z_{3} \times Z_{2 t}\right)$ GBRD.

\subsection{Multiples of Twelve}

Theorem 32. Let $G=Z_{3} \times Z_{2 t}$ where $t \geq 2$ is a power of 2 . If $v \geq 4$, then a $(v, 3,6 t ; G)$ GBRD exists.

Proof. Let $K=\{4,5,6,7,8,9,10,11,12,14,15,18,19,23\}$. It suffices to establish the existence of a $(v, 3,|G| ; G)$ GBRD for $v \in K$, since for other $v$ s a $(v, K, 1)$ PBD exists by Table 2, so then we can use Theorem 18 for our result.

If $v \equiv 0,1(\bmod 3)$ and $v>3$, then a $\left(v, 3,2 t ; Z_{2 t}\right)$ GBRD exists by Theorem 30 . Using Theorem 15, we may form the direct product of this GBRD with a $\left(v, 3,3 ; Z_{3}\right)$ GBRD given by Lemma 20. If $v \equiv 0,1(\bmod 4)$, then a $\left(v, 3,2 t ; Z_{2 t}\right)$ GBRD exists by Theorem 31. We may use the $\left(v, 4,6 ; Z_{3} \times Z_{2}\right)$ GBRDs with $v=11,14$ and 23 given in Examples 46 and 47 and the $\left(4,3, t ; Z_{t}\right)$ GBRD of Example 48 in Theorem 16 to give a $\left(v, 3,6 t ; Z_{3} \times Z_{2 t}\right)$ GBRD for these $v$ s.

\section{Non-cyclic Sylow 2-Subgroups}

We have already discussed $v=3$ in Section 6 .

Lemma 33. Let $|G| \geq 4$ be a power of 2 . A $(4,3,|G| ; G)$ GBRD exists if $G$ is non-cyclic.

Proof. Since a $\left(4,3,2 t ; Z_{2 t}\right)$ GBRD exists for any $t \geq 1$, if $G$ has of more than 2 invariants of even order, then we can use Theorem 15 to form the direct product of the $\left(4,3,2 t ; Z_{2 t}\right)$ GBRD and a $(3,3,|H| ; H)$ GBRD where $G=Z_{2 t} \times H$, so we only need consider the case of 2 invariants of even order, say $G=Z_{2 t} \times Z_{2 s}$ with $t \geq s \geq 1$. Now we may use Theorem 15 to combine a $\left(4,3, t ; Z_{t}\right)$ GBRD, which we will treat as a $\left(4,3, t ; Z_{t} \times Z_{1}\right)$ GBRD with a $\left(3,3,2 s ; Z_{2} \times Z_{2 s}\right)$ GBRD. This fails if $t=1$, but then the needed $\left(4,3,4 ; Z_{2} \times Z_{2}\right) \mathrm{GBRD}$ exists by Example 49.

Lemma 34. Let $|G| \geq 4$ be a power of 2 . $A(6,3,|G| ; G) G B R D$ exists if $G$ is non-cyclic.

Proof. Since a $\left(6,3,2 t ; Z_{2 t}\right)$ GBRD exists for any $t \geq 2$, if $G$ consists of 3 or more invariants of even order, then we can use Theorem 15 to form the direct product of the $\left(6,3,2 t ; Z_{2 t}\right)$ GBRD and a $(3,3,|H| ; H)$ GBRD where $G=Z_{2 t} \times H$, unless all the invariants are of order 2, i.e., $G=E A(|G|)$, but this case is constructed by starting with a $\left(6,3,4 ; Z_{2} \times Z_{2}\right) \mathrm{GBRD}$ and then forming the direct product (using Theorem 15) with a $(3,3,|G| / 4 ; E A(|G| / 4))$ 
GBRD which will only fail if $|G| / 4=2$, but in Example 52 we give a direct construction of a $(6,3,8 ; E A(8))$ GBRD. So now we only need consider the case of 2 invariants of even order, say $G=Z_{2 t} \times Z_{2 s}$ with $t \geq s \geq 1$. Now we may use Theorem 15 to combine a $\left(6,3, t ; Z_{t}\right)$ GBRD, which we will treat as a $\left(6,3, t ; Z_{t} \times Z_{1}\right)$ GBRD with a $\left(3,3,2 s ; Z_{2} \times Z_{2 s}\right)$ GBRD. This fails if $t \leq 2$, so we need to construct $(6,3,|G| ; G)$ GBRDs with $G=Z_{2} \times Z_{2}$, $G=Z_{2} \times Z_{4}$ and $G=Z_{4} \times Z_{4}$. Now the first two of these GBRDs were given in Example 52 . A $\left(6,3,16 ; Z_{4} \times Z_{4}\right)$ GBRD may be constructed from a $\left(6,3,4 ; Z_{2} \times Z_{2}\right)$ GBRD and a $\left(3,3,4 ; Z_{2} \times Z_{2}\right)$ GBRD using Theorem 16 .

Theorem 35. Let $|G|=2 t$ where $t \geq 2$ is a power of 2 and the Sylow 2-subgroup of $G$ is non-cyclic. If $v \equiv 0,1(\bmod 3)$, then $a(v, 3,2 t ; G) G B R D$ exists.

Proof. Let $K=\{3,4,6\}$. It suffices to establish the existence of a $(v, 3, G ; G)$ GBRD for $v \in K$, since for other $v$ s a $(v, K, 1)$ PBD exists by Table 2 , so then we can use Theorem 18 for our result.

If $v=3,4$ or 6 , then a $(v, 3,2 t ; G)$ GBRD exists by Theorem 26, Lemmas 33 and 34 .

Theorem 36. Let $|G|=3 t$ where $t \geq 4$ is a power of 2 and the Sylow 2-subgroup of $G$ is non-cyclic. If $v \geq 3$, then a $(v, 3,3 t ; G) G B R D$ exists.

Proof. Let $K=\{3,4,5,6,8\}$ and $G=Z_{3} \times H$. It suffices to establish the existence of a $(v, 3, G ; G)$ GBRD for $v \in K$, since for other $v$ s a $(v, K, 1)$ PBD exists by Table 2 , so then we can use Theorem 18 for our result.

If $v=3,4$ or 6 , then a $(v, 3, t ; H)$ GBRD exists by Theorem 35 . Using Theorem 15 , we may form the direct product of this GBRD with a $\left(3,3,3 ; Z_{3}\right)$ GBRD given by Lemma 20 . If $v=5$ or 8 , then a $\left(v, 4,3 ; Z_{3}\right)$ GBRD exists by Examples 10 and 45 . Using Theorem 15, we may form the direct product of this GBRD with a $(4,3,|H| ; H)$ GBRD given by Lemma 33.

\section{Sufficiency}

We will divide our proof of sufficiency of the necessary conditions (namely (1), (2), (3) and (4)) into the cases $\lambda=|G|$ and $\lambda>|G|$.

Theorem 37. The necessary conditions for the existence of a $(v, 3, \lambda ; G)$ GBRD are sufficient when $\lambda=|G|$.

Proof. We start with a $(v, 3,|H| ; H)$ GBRD for some normal subgroup $H \subset G$.

Suppose $|G|$ is not divisible by 3 . If $|G|$ is also not divisible by 2 , we start with a $(v, 3,1)$ BIBD, noting that if we sign every element in every block with 0 , this can be considered as a $\left(v, 3,1 ; Z_{1}\right)$ GBRD where $Z_{1}$ is the trivial group. If $|G|$ is also divisible by 2 , our starting design depends on the number of even invariants. If there is only one, and it is $Z_{2}$, then we start with the $\left(v, 3,2 ; Z_{2}\right)$ GBRD given by Theorem 29 , and if it is $Z_{4 t}$ for some $t \geq 1$, then we start with the $\left(v, 3,4 t ; Z_{4 t}\right)$ GBRD given by Theorem 30 . If there are two or more, then we start with the $(v, 3, H ; H)$ GBRD given by Theorem 35 , where $H$ is the direct product of all the even invariants. Using Theorem 15 with this initial GBRD, we may successively form the direct products with the $\left(3,3, n ; Z_{n}\right)$ GBRD given by Lemma 20 for each odd invariant in $G / H$.

Now suppose $|G|$ is divisible by 3 . If $|G|$ is also not divisible by 2 , we start with a $\left(v, 3,3 ; Z_{3}\right)$ GBRD given by Theorem 23 . If $|G|$ is also divisible by 2 , our starting design 
depends on the number of even invariants. If there is only one, and it is $Z_{2}$, then we start with the $\left(v, 3,6 ; Z_{3} \times Z_{2}\right)$ GBRD given by Theorem 31, and if it is $Z_{4 t}$ for some $t \geq 1$, then we start with the $\left(v, 3,12 t ; Z_{3} \times Z_{4 t}\right)$ GBRD given by Theorem 32 . If there are two or more, then we start with the $\left(v, 3,3 F ; Z_{3} \times F\right)$ GBRD given by Theorem 36 , where $F$ is the direct product of all the even invariants. Now consider some fixed invariant that is divisible by 3 . If this invariant has order $3 s$ with $s \geq 3$, then we may apply Theorem 16 using the $\left(v, 3, s ; Z_{s}\right)$ GBRD given by Lemma 20 with our starting GBRD, to give a $\left(v, 3,3 s|F| ; Z_{3 s} \times F\right)$ GBRD. So we take $H=Z_{3 s} \times F$ as our starting GBRD, then using Theorem 15 and this GBRD, we may successively form the direct products with the $\left(3,3, n ; Z_{n}\right)$ GBRD given by Lemma 20 for each odd invariant in $G / H$.

Having shown the necessary conditions for the existence of a $(v, 3,|G| ; G)$ GBRD are sufficient for all finite Abelian groups $G$, we can exploit Theorem 5 to solve many of the cases where $\lambda>|G|$.

Theorem 38. If $G$ is finite Abelian, then the necessary conditions for the existence of a $(v, 3, \lambda ; G)$ GBRD are sufficient.

Proof. We know from Theorem 37 that the necessary conditions are sufficient for the existence of a $(v, 3, \lambda ; G)$ GBRD if $\lambda=|G|$, so we now need to consider $\lambda=n|G|$ with $n>1$.

If $\lambda \not \equiv 2(\bmod 4)$, then the necessary conditions for a $(v, 3, \lambda)$ BIBD coincide with those for a $(v, 3, n|G| ; E A(n) \times G)$ GBRD for $v>3$, so we may take this GBRD and contract it using Theorem 5 to obtain a $(v, 3, n|G| ; G)$ GBRD for $v>3$. If $v=3$ and $\lambda \not \equiv 2(\bmod 4)$, this construction also works except in the case that $\lambda$ is odd and $G$ has a cyclic Sylow 2-subgroup, when no $(3,3, n|G| ; G)$ GBRD exists by Theorem 3.

If $\lambda \equiv 2(\bmod 4)$ and $n$ is odd, the necessary conditions for a $(v, 3, n|G| ; G)$ GBRD coincide with those for a $(v, 3, n|G| ; E A(n) \times G)$ GBRD for $v>3$, so we may take this latter GBRD and contract it using Theorem 5 to obtain a $(v, 3, n|G| ; G)$ GBRD.

So now we must consider the case that $n \equiv 2(\bmod 4)$ and $|G|$ is odd. If $|G| \equiv 1,5$ $(\bmod 6)$, we can take a $(v, 3, n)$ BIBD and a $(3,3,|G| ; G)$ GBRD and apply Theorem 15 for our solution. So now we only need to consider $|G| \equiv 3(\bmod 6)$, with $n \equiv 2(\bmod 4)$, and we can simply juxtapose $n / 2$ copies of a $(v, 3,2|G| ; G)$ GBRD. It only remains to exhibit this $(v, 3,2|G| ; G)$ GBRD. For $v=3$ or 5 , we can take two copies of the $(v, 3,|G| ; G)$ GBRD; for $v=4$ or 6 , we can take a $(v, 3,2)$ BIBD and a $(3,3,|G| ; G)$ GBRD and apply Theorem 15 for the $(v, 3,2|G| ; G)$ GBRD and for $v=8$, we can take a $\left(v, 3,2|G| ; Z_{2} \times G\right)$ GBRD and apply Theorem 5. Now we can use these GBRDs in Theorem 18 to break the blocks of the $(v,\{3,4,5,6,8\}, 1)$ PBD given by Table 2 .

Acknowledgements. The research of the first author is supported by National Natural Science Foundation of China under Grant No. 10471127 and Zhejiang Provincial Natural Science Foundation of China under Grant No. R604001.

\section{References}

1. Abel, R.J.R., Bennett, F.E., Ge, G.: The existence of four HMOLS with equal sized holes. Des. Codes Cryptogr. 26, 7-31 (2002).

2. Abel, R.J.R., Combe, D., Palmer, W.D.: Bhaskar Rao designs and the groups of order 12. Australas. J. Combin. 29, 301-308 (2004). 
3. Abel, R.J.R., Combe, D., Palmer, W.D.: Generalized Bhaskar Rao designs and dihedral groups. J. Combin. Theory Ser. A 106, 145-157 (2004).

4. Bennett, F.E., Gronau, H-D.O.F., Ling, A.C.H., Mullin, R.C.: PBD-closure. In: C.J. Colbourn and J.H. Dinitz: The CRC Handbook of Combinatorial Designs, pp. 203-213 CRC Press, Boca Raton, FL, 1996.

5. Beth, T., Jungnickel, D., Lenz, H.: Design Theory Cambridge University Press, Cambridge, UK, 1999.

6. Bose, R.C.: An affine analogue of Singer's theorem. J. Indian Math. Soc. 6, 1-15 (1942).

7. Bowler, A., Quinn, K., Seberry, J.: Generalized Bhaskar Rao Designs with elements from cyclic groups of even order. Australas. J. Combin. 3, 5-13 (1991).

8. Brouwer, A.E., Schrijver, A., Hanani, H.: Group divisible designs with block-size four. Discrete Math. 20, 1-10 (1977).

9. Combe, D., Palmer, W.D., Unger, W.R.: Bhaskar Rao Designs and the Alternating Group $A_{4}$. Australas. J. Combin. 24, 275-283 (2001).

10. de Launey, W.: Bhaskar Rao designs, In: C.J. Colbourn and J.H. Dinitz: The CRC Handbook of Combinatorial Designs, pp. 241-246 CRC Press, Boca Raton, FL, 1996.

11. de Launey, W., Sarvate, D.G., Seberry, J.: Generalized Bhaskar Rao Designs with block size 3 over $Z_{4}$. Ars Combin. 19A, 273-285 (1985).

12. de Launey, W., Seberry, J.: On generalized Bhaskar Rao designs of block size four. Congr. Numer. 41, 229-294 (1984).

13. Drake, D.A.: Partial $\lambda$-geometries and generalized Hadamard matrices over groups. Canad. J. Math. 31, 617-627 (1979).

14. Gallant, R.P., Jiang, Z., Ling, A.C.H.: The spectrum of cyclic group divisible designs with block size three. J. Combin. Des. 7, 95-105 (1999).

15. Gibbons, P.B., Mathon, R.: Construction methods for Bhaskar Rao and related designs. J. Austral. Math. Soc. Ser. A 42, 5-30 (1987); ibid. 43, 420 (1987).

16. Hall, M. Jr., The Theory of Groups Macmillan, New York, 1959.

17. Hanani, H.: Balanced incomplete block designs and related designs. Discrete Math. 11, 255-369 (1975).

18. Jiang, Z.: Concerning cyclic group divisible designs with block size three. Australas. J. Combin. 13, 227-245 (1996).

19. Lam, C., Seberry, J.: Generalized Bhaskar Rao designs. J. Statist. Plann. Inference 10, 83-95 (1984).

20. Mullin, R.C., Ling, A.C.H., Abel, R.J.R., Bennett, F.E.: On the closure of subsets of $\{4,5, \ldots, 9\}$ which contain $\{4\}$. Ars Combin. 45, 33-76 (1997).

21. Palmer, W.D.: A composition theorem for generalized Bhaskar Rao designs. Australas. J. Combin. 6, 221-228 (1992). 
22. Palmer, W., Seberry, J.: Bhaskar Rao designs over small groups. Ars Combin. 26A, 125-148 (1988).

23. Robinson, D.J.S.: A Course in the Theory of Groups Springer-Verlag, New York, 1982.

24. Seberry, J.: Regular group divisible designs and Bhaskar Rao designs with block size three. J. Statist. Plann. Inference 10, 69-82 (1984).

25. Seberry, J.: Generalized Bhaskar Rao designs of block size three. J. Statist. Plann. Inference 11, 273-279 (1985).

26. Seberry, J.: Bhaskar Rao designs of block size three over groups of order 8. Technical Report CC88/4, Dept. of Computer Science, University of N.S.W., Sydney, 1988.

27. Seberry, J.: Bose's method of differences applied to construct Bhaskar Rao designs. J. Statist. Plann. Inference 73, 215-224 (1998).

28. Shen, H.: Constructions and uses of labelled resolvable designs. Lecture Notes in Pure and Appl. Math. 126, 3-14 (1990).

29. Street, D.J., Rodger, C.A.: Some results on Bhaskar Rao designs. Lecture Notes in Math. 829, 238-245 (1980).

\section{Appendix. Some Small Designs}

\section{Difference Matrices}

Note that a $(k, k, \lambda ; G)$ GBRD can be obtained from the signed incidence matrix of a $(k+1, k+1, \lambda ; G)$ GBRD by deleting any row (i.e., point). Also the signed incidence matrix of a $(k, k, \lambda ; G)$ GBRD is often known as a $(|G|, k, \lambda /|G| ; G)$ difference matrix.

Example 39. A $\left(4,4,4 ; Z_{2} \times Z_{2}\right)$ GBRD on $I_{4}$ is given as a difference matrix.

$\begin{array}{llll}00 & 00 & 00 & 00 \\ 00 & 01 & 10 & 11 \\ 00 & 10 & 11 & 01 \\ 00 & 11 & 01 & 10\end{array}$

A $\left(4,4,8 ; Z_{2} \times Z_{4}\right)$ GBRD on $I_{4}$ is given as a difference matrix.

$\begin{array}{llllllll}00 & 00 & 00 & 00 & 00 & 00 & 00 & 00 \\ 00 & 01 & 02 & 03 & 10 & 11 & 12 & 13 \\ 00 & 03 & 13 & 12 & 11 & 10 & 02 & 01 \\ 00 & 12 & 03 & 11 & 13 & 01 & 10 & 02\end{array}$

A $\left(4,4,8 ; Z_{2} \times Z_{2} \times Z_{2}\right)$ GBRD on $I_{4}$ is given as a difference matrix.

$\begin{array}{llllllll}000 & 000 & 000 & 000 & 000 & 000 & 000 & 000 \\ 000 & 001 & 010 & 100 & 011 & 110 & 111 & 101 \\ 000 & 010 & 100 & 011 & 110 & 111 & 101 & 001 \\ 000 & 100 & 011 & 110 & 111 & 101 & 001 & 010\end{array}$

Example 40. A $\left(Z_{8}, Z_{2} ; 4\right) \mathrm{HDM}$. 


$\begin{array}{llllll}0 & 0 & 0 & 0 & 0 & 0 \\ 1 & 2 & 3 & 5 & 6 & 7 \\ 6 & 3 & 2 & 7 & 1 & 5 \\ 7 & 1 & 5 & 6 & 3 & 2\end{array}$

Some more general holey difference matrices were given in [7].

Example 41. (Bowler et al. [7]) Let $t \geq 2$. A $\left(Z_{4 t}, Z_{2} ; 3\right)$ HDM in the form $(0, i, f(i))^{T}$ (with $i \notin\{0,2 t\}$ ).

$$
f(i)= \begin{cases}4 t-i-1 & \text { for } 0<i<t \\ t-1 & \text { for } i=t \\ 4 t-i & \text { for } t<i<3 t \quad \text { with } i \neq 2 t \\ 4 t-i-3 & \text { for } 3 t \leq i<4 t \text { with } i-t \text { even } \\ 4 t-i+1 & \text { for } 3 t<i<4 t \text { with } i-t \text { odd }\end{cases}
$$

The smallest example is with $t=2$ (and we cannot add another row here).

$\begin{array}{llllll}0 & 0 & 0 & 0 & 0 & 0 \\ 1 & 2 & 3 & 5 & 6 & 7 \\ 6 & 1 & 5 & 3 & 7 & 2\end{array}$

A direct generalization of Example 40 is given in the next example.

Example 42. For $t \equiv 2,4(\bmod 6)$, an example of a $\left(Z_{4 t}, Z_{t} ; 3\right) \mathrm{HDM}$ in the form $(0, i, f(i))^{T}$ (with $i \notin\{0, t, 2 t, 3 t\}$ ) is given as a holey quasi-difference matrix of the form $(0, i, f(i))^{T}$.

$$
f(i)= \begin{cases}4 t-2 i & \text { for } 0<i<2 t \text { with } i \text { odd } \\ 6 t-i & \text { for } 2 t<i<4 t \text { with } i \text { odd } \\ 2 t-i / 2 & \text { for } 0<i<4 t \text { with } i \equiv 2(\bmod 4)\end{cases}
$$

We can also expand Example 41.

Example 43. A $\left(Z_{2} \times Z_{4 t}, Z_{2} \times Z_{2} ; 3\right)$ HDM: we will use $f(i)$ as defined in Example 41, and take $i=1,2, \ldots, 2 t-1$.

$$
\begin{array}{cccc}
(0,0) & (0,0) & (0,0) & (0,0) \\
(0, i) & (0, i+2 t) & (1, i) & (1, i+2 t) \\
(0, f(i)) & (1, f(i)) & (0, f(i+2 t)) & (1, f(i+2 t))
\end{array}
$$

Block Size 4

Example 44 . A $\left(7,4,2 ; Z_{2}\right)$ GBRD on $Z_{7}$ is given by

$$
\left(0_{0}, 1_{1}, 2_{1}, 4_{1}\right) \text {. }
$$

Example 45. (de Launey and Seberry [12]) An $\left(8,4,3 ; Z_{3}\right)$ GBRD on $Z_{7} \cup\{\infty\}$ is given by

$$
\left(\infty_{0}, 0_{0}, 1_{1}, 3_{2}\right), \quad\left(0_{0}, 1_{0}, 2_{2}, 5_{0}\right) .
$$

A $\left(9,4,3 ; Z_{3}\right)$ GBRD on $Z_{3} \times Z_{3}$ is given by

$$
\left(00_{0}, 01_{0}, 10_{0}, 11_{1}\right), \quad\left(00_{0}, 01_{2}, 12_{1}, 22_{0}\right) .
$$


A $\left(12,4,3 ; Z_{3}\right)$ GBRD on $Z_{11} \cup\{\infty\}$ is given by

$$
\left(\infty_{0}, 0_{0}, 1_{1}, 3_{2}\right), \quad\left(0_{0}, 1_{0}, 3_{0}, 7_{1}\right), \quad\left(0_{0}, 1_{2}, 4_{0}, 6_{2}\right) .
$$

Example 46. (de Launey and Seberry [12]) A $\left(14,4,6 ; Z_{6}\right)$ GBRD on $Z_{13} \cup\{\infty\}$ is given by

$$
\begin{array}{llll}
\left(0_{0}, 1_{0}, 2_{2}, 6_{0}\right), & \left(0_{0}, 1_{3}, 5_{5}, 8_{5}\right), & \left(0_{0}, 1_{5}, 3_{5}, 6_{2}\right), & \left(0_{0}, 2_{3}, 6_{3}, 8_{2}\right), \\
\left(\infty_{0}, 0_{0}, 1_{1}, 3_{2}\right), & \left(\infty_{0}, 0_{3}, 3_{4}, 12_{5}\right), & \left(0_{0}, 2_{4}, 5_{2}, 9_{3}\right) . &
\end{array}
$$

Example 4\%. (Brouwer et al. [8]) Let $v \equiv 3 \bmod 4$ be a prime power with $v>3$. A $\left(v, 4,6 ; Z_{6}\right)$ GBRD may be constructed on the point set $G F(v)$. Let $x$ be a primitive generator, and let $\alpha$ be chosen so that $x^{2 \alpha}-1$ is not a square; (exactly one of $\alpha=1$ or $\alpha=q-2$ will work since $x^{2}-1=-x^{2}\left(x^{-2}-1\right)$.) Our base blocks are:

$$
\left(0_{0}, x_{3}^{\alpha+2 i}, x_{2}^{2 i},-x_{2}^{2 i}\right) \quad \text { for } i=0,1, \ldots,(q-3) / 2 .
$$

\section{Block Size 3}

Example 48. (Gallant et al. [14, pp. 101]) A $\left(4,3,2 t ; Z_{2 t}\right)$ GBRD for any $t \geq 1$. The design on $I_{4}$ is given by

$$
\left(0_{0}, 1_{i}, 2_{2 i}\right), \quad\left(0_{0}, 1_{t+i}, 3_{t-i}\right), \quad\left(0_{0}, 2_{2 i+1}, 3_{t+i+1}\right), \quad\left(1_{0}, 2_{t+i}, 3_{2 i+1}\right),
$$

where $i=0,1, \ldots, t-1$.

Example 49. A $\left(4,3,4 ; Z_{2} \times Z_{2}\right)$ GBRD.

$\begin{array}{llllllll}00 & 00 & 00 & 00 & 00 & 00 & & \\ 01 & 11 & 00 & 10 & & & 10 & 00 \\ 11 & 00 & & & 01 & 10 & 11 & 00 \\ & & 01 & 00 & 10 & 11 & 01 & 00\end{array}$

Example 50. A $\left(5,3,3 ; Z_{3}\right)$ GBRD on $Z_{5}$ is given by

$$
\left(0_{0}, 1_{1}, 4_{1}\right), \quad\left(0_{0}, 2_{1}, 3_{1}\right) \text {. }
$$

Example 51. (Bowler et al. [7, Theorem 2.6]) A $\left(6,3,2 t ; Z_{2 t}\right)$ GBRD for any even $t \geq 2$. The design on $I_{6}$ is given by

$$
\begin{array}{ccc}
\left(0_{0}, 1_{i}, 4_{2 i}\right), & \left(1_{0}, 2_{i}, 5_{2 i}\right), & \left(2_{i}, 3_{2 i}, 0_{0}\right), \\
\left(3_{2 i+1}, 4_{t+i}, 1_{0}\right), & \left(4_{i+1}, 5_{2 t-i-1}, 2_{0}\right), & \left(1_{0}, 2_{t+i}, 3_{2 i}\right), \\
\left(3_{0}, 4_{t+i}, 5_{2 t-i-1}\right), & \left(5_{t-i-1}, 0_{0}, 1_{t+i}\right), & \left(0_{0}, 2_{t+i}, 4_{2 i+1}\right), \\
& \left(0_{0}, 3_{2 i+1}, 5_{t+i}\right), &
\end{array}
$$

where $i=0,1, \ldots, t-1$.

Example 52. A $\left(6,3,4 ; Z_{2} \times Z_{2}\right)$ GBRD on $Z_{5} \cup\{\infty\}$.

$$
\left(\infty_{00}, 0_{00}, 1_{01}\right), \quad\left(\infty_{00}, 0_{10}, 2_{11}\right), \quad\left(0_{00}, 1_{00}, 4_{10}\right), \quad\left(0_{00}, 2_{00}, 3_{11}\right) .
$$

A $\left(6,3,8 ; Z_{2} \times Z_{2} \times Z_{2}\right)$ GBRD on $Z_{5} \cup\{\infty\}$.

$\left(\infty_{000}, 0_{000}, 1_{010}\right),\left(\infty_{000}, 0_{100}, 2_{110}\right),\left(0_{000}, 1_{001}, 4_{111}\right),\left(0_{000}, 2_{000}, 3_{011}\right)$, $\left(\infty_{000}, 0_{001}, 1_{101}\right),\left(\infty_{000}, 0_{011}, 2_{111}\right),\left(0_{000}, 1_{000}, 4_{101}\right),\left(0_{000}, 2_{001}, 3_{111}\right)$.

A $\left(6,3,8 ; Z_{2} \times Z_{4}\right)$ GBRD on $Z_{5} \cup\{\infty\}$. 


$$
\begin{array}{llll}
\left(\infty_{00}, 0_{00}, 1_{01}\right), & \left(\infty_{00}, 0_{10}, 2_{12}\right), & \left(0_{00}, 1_{11}, 4_{00}\right), & \left(0_{00}, 2_{00}, 3_{10}\right), \\
\left(\infty_{00}, 0_{11}, 1_{13}\right), & \left(\infty_{00}, 0_{03}, 2_{02}\right), & \left(0_{00}, 1_{13}, 4_{12}\right), & \left(0_{00}, 2_{12}, 3_{11}\right) .
\end{array}
$$

Example 53. A $\left(9,3,2 n ; Z_{2 n}\right)$ GBRD missing a $\left(4,3,2 n ; Z_{2 n}\right)$ GBRD subdesign on the first

\begin{tabular}{|c|c|c|c|c|c|c|c|c|c|c|c|c|c|c|c|c|c|c|c|}
\hline e & e & e & e & e & & & & & & & & & & & & & & & \\
\hline & & & & & e & e & e & e & $\mathrm{e}$ & & & & & & & & & & \\
\hline & & & & & & & & & & e & $\mathrm{e}$ & $\mathrm{e}$ & e & e & $\mathrm{e}$ & $\mathrm{e}$ & e & e & $\mathrm{e}$ \\
\hline $\mathrm{x}$ & $\mathrm{S}$ & & & & $\mathrm{x}$ & $\mathrm{s}$ & & & & $\mathrm{x}$ & $\mathrm{S}$ & & & & $\mathrm{x}$ & $\mathrm{S}$ & & & \\
\hline \multirow[t]{4}{*}{$\mathrm{y}$} & & $\mathrm{s}$ & & & & $\mathrm{y}$ & $\mathrm{S}$ & & & & & $\mathrm{x}$ & $\mathrm{S}$ & & & & $\mathrm{X}$ & $\mathrm{S}$ & \\
\hline & $\mathrm{y}$ & & $\mathrm{S}$ & & & & $\mathrm{y}$ & $\mathrm{S}$ & & $\mathrm{y}$ & & & & $\mathrm{S}$ & & & & $\mathrm{t}$ & $\mathrm{X}$ \\
\hline & & $\mathrm{t}$ & & $\mathrm{x}$ & & & & $t$ & $\mathrm{X}$ & & $\mathrm{t}$ & & $\mathrm{y}$ & & & $\mathrm{y}$ & & & $t$ \\
\hline & & & $\mathrm{y}$ & $\mathrm{t}$ & $\mathrm{t}$ & & & & $\mathrm{y}$ & & & $\mathrm{y}$ & & $\mathrm{t}$ & $\mathrm{y}$ & & $\mathrm{t}$ & & \\
\hline
\end{tabular}
4 points for any odd $n \geq 1$. Consider the following signed incidence matrix.

Now take $e=0$ and $x=i$ for $i=0,1, \ldots, n-1, y=2 i$ for $i=0,1, \ldots,(n-1) / 2$ with $y=2 i-n$ for $i=(n+1) / 2, \ldots, n-1$ if $n>1$. Finally take $s=n+x$ and $t=n+y$.

Example 54 . A $\left(9,3,4 ; Z_{4}\right)$ GBRD over $Z_{9}$ is given by

$$
\left(0_{0}, 1_{1}, 3_{3}\right), \quad\left(0_{0}, 1_{2}, 3_{1}\right), \quad\left(0_{0}, 1_{3}, 5_{1}\right), \quad\left(0_{0}, 2_{1}, 5_{3}\right) .
$$

Finally we adjoin the blocks of a $(9,3,1)$ BIBD signed uniformly with 0 .

Example 55. (Gallant et al. [14]) A $\left(10,3,4 ; Z_{4}\right)$ GBRD given as a $(4,1)$ GDD of type $4^{10}$ over $Z_{40}$. This GDD can be converted into the GBRD using the process described in Example 11.

$$
(0,1,9), \quad(0,2,16), \quad(0,3,18), \quad(0,4,11), \quad(0,5,17), \quad(0,6,19) .
$$

Example 56 . A $\left(15,3,4 ; Z_{4}\right)$ GBRD over $Z_{15}$ is given by

$$
\begin{array}{llll}
\left(0_{0}, 1_{1}, 6_{2}\right), & \left(0_{0}, 1_{2}, 7_{3}\right), & \left(0_{0}, 1_{3}, 4_{2}\right), & \left(0_{0}, 2_{1}, 5_{2}\right), \\
\left(0_{0}, 2_{2}, 6_{3}\right), & \left(0_{0}, 2_{3}, 7_{2}\right), & \left(0_{0}, 3_{2}, 7_{1}\right) . &
\end{array}
$$

Finally we adjoin the blocks of a $(15,3,1)$ BIBD signed uniformly with 0 .

Example 5\%. Our $\left(15,3,8 ; Z_{8}\right)$ GBRD is actually formed on the points $\left(I_{2} \times Z_{7}\right) \cup\{\infty\}$, but we present it on the points $Z_{14} \cup\{\infty\}$ where the development is with the order 7 automorphism $x \mapsto x+2$.

$$
\begin{array}{cccc}
\left(2_{0}, 3_{0}, 4_{0}\right), & \left(2_{0}, 3_{1}, 8_{0}\right), & \left(2_{0}, 3_{2}, 8_{2}\right), & \left(2_{0}, 4_{1}, 11_{2}\right), \\
\left(3_{0}, 4_{1}, 6_{0}\right), & \left(2_{0}, 9_{0}, 10_{2}\right), & \left(2_{0}, 5_{0}, 7_{0}\right), & \left(2_{0}, 5_{1}, 7_{2}\right), \\
\left(2_{0}, 5_{2}, 7_{1}\right), & \left(2_{0}, 5_{3}, 7_{5}\right), & \left(2_{0}, 5_{4}, 7_{7}\right), & \left(2_{0}, 5_{5}, 7_{3}\right), \\
\left(2_{0}, 5_{7}, 7_{4}\right), & \left(2_{0}, 5_{6}, 10_{1}\right), & \left(2_{0}, 7_{6}, 13_{1}\right), & \left(3_{0}, 5_{4}, 11_{0}\right), \\
\left(2_{0}, 9_{2}, 13_{2}\right), & \left(3_{0}, 7_{1}, 13_{3}\right), & \left(3_{0}, 6_{1}, 10_{2}\right), & \left(2_{0}, 6_{0}, 10_{4}\right), \\
\left(2_{0}, 6_{2}, 12_{5}\right), & \left(3_{0}, 7_{4}, 13_{1}\right), & \left(2_{0}, 8_{5}, 12_{2}\right), & \left(2_{0}, 6_{7}, 13_{3}\right), \\
\left(2_{0}, 3_{3}, 4_{6}\right), & \left(2_{0}, 3_{4}, 4_{3}\right), & \left(2_{0}, 3_{5}, 4_{2}\right), & \left(2_{0}, 3_{7}, 4_{5}\right), \\
\left(2_{0}, 3_{6}, 13_{4}\right), & \left(2_{0}, 9_{3}, 13_{6}\right), & \left(2_{0}, 9_{7}, 13_{5}\right), & \left(2_{0}, 9_{5}, 1_{4}\right), \\
\left(\infty_{0}, 2_{3}, 4_{7}\right), & \left(\infty_{0}, 2_{0}, 11_{3}\right), & \left(\infty_{0}, 2_{1}, 11_{0}\right), & \left(\infty_{0}, 3_{1}, 9_{2}\right), \\
\left(\infty_{0}, 5_{4}, 1_{6}\right), & \left(\infty_{0}, 7_{6}, 12_{2}\right), & \left(\infty_{0}, 1_{7}, 7_{5}\right), & \left(\infty_{0}, 2_{4}, 8_{5}\right) .
\end{array}
$$

Example 58. A $\left(21,3,2 ; Z_{2}\right)$ GBRD missing a $\left(4,3,2 ; Z_{2}\right)$ GBRD subdesign (on the 4 letters) with a point set of $Z_{17} \cup\{A, B, C, D\}$ is given by 


$$
\begin{array}{llll}
\left(A_{0}, 0_{0}, 2_{1}\right), & \left(B_{0}, 0_{0}, 6_{1}\right), & \left(C_{0}, 0_{0}, 7_{1}\right), & \left(D_{0}, 0_{0}, 8_{1}\right) \\
\left(0_{0}, 1_{0}, 3_{0}\right), & \left(0_{0}, 1_{1}, 5_{0}\right), & \left(0_{0}, 3_{1}, 8_{0}\right), & \left(0_{0}, 4_{0}, 10_{0}\right) .
\end{array}
$$

Example 59. For a $\left(27,3,4 ; Z_{4}\right)$ GBRD, let $x$ be a root of $x^{3}=x^{2}+2$ in $G F(27)$. Generate 13 base blocks be repeatedly multiplying $\left(0_{0}, 1_{1},\left(x^{2}+2 x+2\right)_{3}\right)$ by $x^{2}$. Note that $1=x^{0}$, $x^{2}+2 x+2=x^{4}$ and $x^{2}+2 x+1=x^{10}$. Develop these blocks over $G F(27)$, then adjoin the blocks of a $(27,3,1)$ BIBD uniformly signed with 0 .

Example 60. A $\left(27,3,8 ; Z_{8}\right)$ GBRD on $Z_{17} \cup\left\{\infty_{t}: t=1, \ldots, 10\right\}$ missing a $\left(10,3,8 ; Z_{8}\right)$ GBRD subdesign on the infinite points.

The base blocks are $\left(0_{3}, i_{0},(17-i)_{0}\right)$ for $i=1,2, \ldots, 8$, plus 5 sets of the form $\left(0_{a},(4 j+\right.$ $\left.1)_{b}\right),\left(0_{c},(4 j+2)_{d}\right),\left(0_{e},(4 j+3)_{f}\right)$ and $\left(0_{g},(4 j+4)_{h}\right)$ for $j=0,1$. Each of the last 5 sets for $j=0$ and the 5 sets for $j=1$ form a signed parallel class (so we can augment these 10 sets with an infinite element of arbitrary, but fixed, signing) provided we make suitable choices for $a, b, \ldots, h$. We can make the same 5 choices for $j=0$ and $j=1$; the 5 choices we make are:

$$
\begin{gathered}
(a, b ; c, d ; e, f ; g, h)=(0,1 ; 2,3 ; 4,5 ; 6,7), \quad(1,0 ; 3,2 ; 5,4 ; 7,6) \\
(0,2 ; 1,3 ; 4,6 ; 5,7), \quad(2,0 ; 3,1 ; 6,4 ; 7,5) \text { and }(0,4 ; 1,5 ; 2,6 ; 3,7) .
\end{gathered}
$$

